\title{
SUPERGONDUGTING SHIELDING
}

\author{
By W. O. HAMILTON, \\ Stanford University, Department of Physics, Stanford, California (U.S.A.).
}

\begin{abstract}
Superconducting shields offer the possibility of obtaining truly zero magnetic fields due to the phenomenon of flux quantization. They also offer excellent shielding from external time varying fields. Various techniques of superconducting shielding will be surveyed and recent results discussed.
\end{abstract}

I. Introduction. - Superconductivity has been a field of active research interest since its discovery in 1911. Many of the possible uses of superconductivity have been apparent from that time but it has been only recently that science and technology have progressed to the point that it has become absolutely necessary to use superconductors for important and practical measurements which are central to disciplines other than low temperature physics. The various superconducting magnetometers which are now being used in many fields, high field superconducting magnets, superconducting linear accelerators have all opened new research areas and have made the use of low temperature phenomena and techniques even more available. We will discuss the use of superconducting materials for shielding against electrical and magnetic fields. In many measurements, the use of superconducting shielding provides the only practical solution for shielding large volumes from fluctuating fields and it provides the only way to obtain a large region of truly zero magnetic field.

II. The Phenomenon of Superconductivity. Superconductivity is observed in many metals when they are cooled to the temperature of liquid helium, $4.2 \mathrm{oK}$. The most striking physical effect seen when a material goes into the superconducting state is the appearance of zero resistance. Superconducting rings of tin have been made to carry currents of an amp by pulling a permanent magnet out of the ring after it has become superconducting. These currents have persisted for periods up to a year without any measurable decrease.

This superconducting state can be destroyed by the application of a magnetic field. If the resistance of a superconductor is measured by a weak current and a magnetic field is applied perpendicular to the superconducting surface a very sharp transition to a normal state is observed when the field is increased. This field is known as the critical field and it is almost a constant at temperatures well below the superconducting transition temperature. The critical field approaches zero as the temperature is raised to approach the superconducting transition temperature. This behavior is shown in figure 1.

The existence of the critical field limits the size of the currents which can be transported by the superconductor. When the current density is increased to the point that the magnetic field created by the

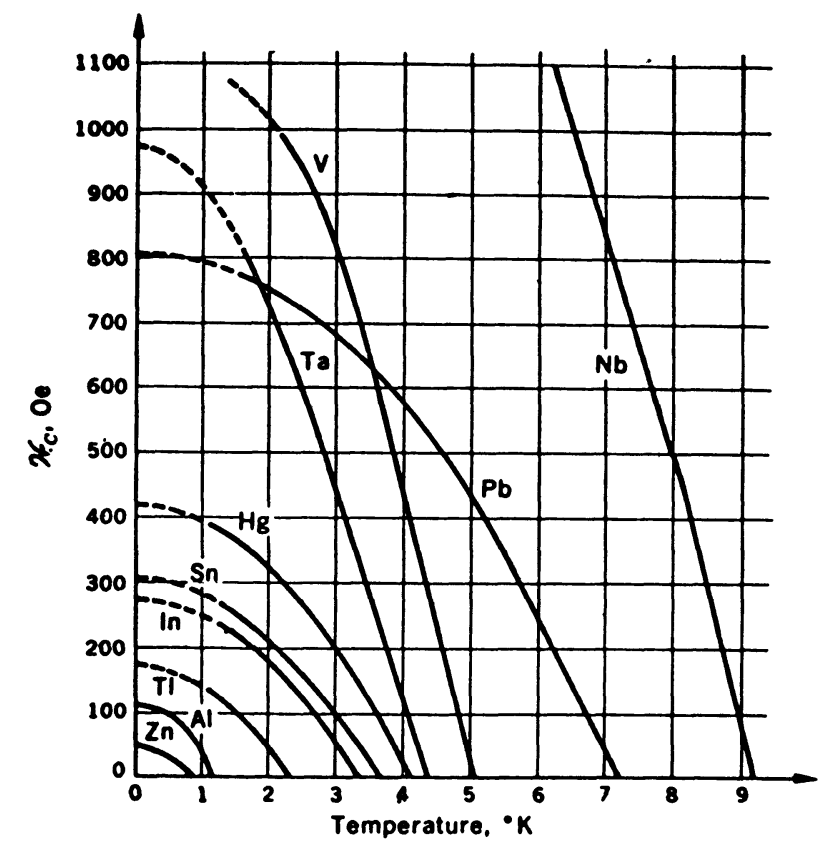

FIG. 1.

current at the surface of the superconductor equals the critical field the material reverts to the normal state, resistance appears and the material behaves like an ordinary electrical conductor.

It was thought for many years that the superconducting state could be adequately described by an infinite conductivity. Indeed this is all one need consider for several types of superconducting shielding. However a look at Maxwell's equations is helpful to understand how a superconductor differs from merely a perfect conductor. Maxwell's equation tells us :

$$
\nabla \times \mathbf{E}=\frac{1}{c} \frac{\partial \mathbf{B}}{\partial t}
$$

and :

$$
\mathbf{E}=\frac{\mathbf{J}}{\sigma}
$$

which is Ohm's law. If $\mathbf{E}$ is required to be zero by Ohm's law due to $\sigma$ being infinite, then Maxwell's equation says that $\mathbf{B}$ must be constant in time. Thus if a changing external magnetic field is incident on a superconductor the superconductor should develop screening currents which oppose the change in $\mathbf{B}$. Since the material has no resistance, the screening 
currents do not die out and the $B$ field in the metal remains constant. The applications of this to shielding are obvious and will be discussed in more detail later.

In 1933, Meissner examined the magnetic field close to a superconducting body and found that when tin becomes superconducting it tends to exclude the magnetic field which was penetrating the metal before the superconducting transition took place. This effect, the Meissner effect, cannot be explained simply by assuming perfect conductivity. We would expect the perfectly conducting material to simply trap whatever magnetic field happened to penetrate the material prior to the superconducting transition. Thus Meissner showed that the superconductor also behaves like a perfect diamagnet, tending to exclude all magnetic fields from its interior.

Experimentally it was found that a perfect Meissner effect was extremely difficult to achieve. Most substances tend to exclude some of the applied field when they go superconducting, the rest of the field is then trapped in the material. These regions of trapped magnetic flux are associated with non superconducting inclusions. The perfect conductivity of the material makes it difficult to eliminate the trapped fields.

The problem of explaining the apparent diamagnetism and the perfect conductivity was undertaken by F. and H. London. The results were the famous London equations which do account for the principal phenomena associated with superconductivity and which also suggest a very general quantum mechanical explanation of superconductivity. Central to this interpretation is the concept of a single quantum mechanical state for the entire macroscopic superconductor. From this idea of a single quantum mechanical state arose F. London's suggestion that the trapped magnetic flux might be quantized.

In 1961, Deaver and Fairbank and Doll and Naubauer demonstrated that the magnetic flux which could be trapped in a hollow superconducting cylinder was quantized. The flux quantum was measured to be :

$$
\Phi_{0}=\frac{h c}{2 e}=2 \times 10^{-7} \text { gauss. } \mathrm{cm}^{2} .
$$

The measurements indicate the validity of the London predictions. For any closed path entirely in a superconductor the magnetic flux linked by the path must be an integer multiple of the flux quantum. Theflux then which is observed trapped in the Meissner effect experiments must be quanta of flux which have become surrounded by zero resistance material. We shall see later that this admits the possibility of using the quantization of magnetic flux to completely eliminate external magnetic fields from a experimental region.

III. Magnetic Shielding Against Time Varying Fields. - The existence of infinite conductivity and the Meissner effect immediately suggest the use of superconducting shields to screen an experiment from external fields. The superconductor provides an extremely effective shield against time varying fields. In order to use the superconductor effectively it is necessary to examine briefly the manner in which fields decrease at the surface of the superconductor.

The London equations predict that in the stationary situation the internal magnetic field will decrease exponentially from the surface of the superconductor, the magnetic field being pushed out from the interior of the superconductor by screening currents which flow at the surface. The characteristic length for the exponential fall off is between 370 and $500 \AA$ in lead. The majority of the screening. Meissner currents flow in a surface layer of this thickness. The internal electric field is zero due to the infinite conductivity. For low frequencies then we expect screening currents to flow in the surface sheath of thickness $500 \AA$. These currents will exclude the fields from the bulk of the material and hence will shield an interior cavity from these fields. A small interior electric field also arises in this sheath due to the time varying magnetic field but it is screened from the bulk of the material by the superconducting electrons. Thus we conclude that a superconducting shield which is thick compared to the penetration depth should shield an internal cavity perfectly from slowly time varying fields.

The next consideration to ask is just what does comprise a slowly varying field. The answer is that the superconducting material begins to look like a normal metal when the frequencies of the external fields become high enough that the normal skin depth becomes comparable to the penetration depth. Physically this situation arises when one considers what happens in the superconducting shielding layer. The time varying currents in this sheath create electric fields which lag the currents in phase. This in turn causes losses in the superconductor. In the two fluid model these electric fields act on the normal electrons and create a normal current. At low frequencies these normal currents are very small. When the normal currents become comparable to the superconducting screening currents then the material behaves much like a normal metal. The frequency at which this occurs is approximately $10^{10} \mathrm{~Hz}$ in the London theory. Thus we expect the superconductor to be an extremely efficient shield of time varying fields up to this frequency and from that point on to be no worse than a normal metal.

IV. Shielding of D.C. Fields. - The previous discussion has centered on the elimination of time varying fields. The infinite conductivity of the superconductor is the only property which it has been necessary to use to obtain this shielding. We now wish to examine the Meissner effect to see how it can be used to reduce or completely eliminate the effects of the ambient magnetic field on an experiment.

We discussed earlier the penetration of a d.c. magnetic field into a surface layer of the superconductor. This layer is about $500 \AA$ thick for lead at $4.2 \mathrm{oK}$. Obviously then to be an effective shield the superconductor must be many penetration depths thick. This is not a serious problem unless the experiment dictates that the shield be an evaporated thin film.

If we had a perfect Meissner effect there would be no problem obtaining regions of zero magnetic field. A shield would be made of a superconducting material and the material would be cooled through its transition temperature. The Meissner effect would cause the superconductor to exclude the magnetic flux which penetrated it. The experiment inside the shield would then be in a region of zero magnetic field as long as the field at the surface of the superconductor remained 
below the critical field. A perfect Meissner effect is unfortunately never observed.

We have already seen that the magnetic flux trapped in a hollow superconducting ring must be quantized. This must also be true for the magnetic flux which is not expelled from the bulk of the superconductor. The picture we have is that of a normal region in the superconductor, this region being held normal by currents flowing around it. This current vortex contains an integer number of flux quanta. The magnetic field everywhere in the superconductor is then zero except in the region of the normal core. These normal regions have been observed by Trauble and Essmann ( fig. 2). Inside a cavity which is surrounded by a superconducting shield containing some trapped flux the field will be that created by the current dipole which flows around the core (fig. 3 ).

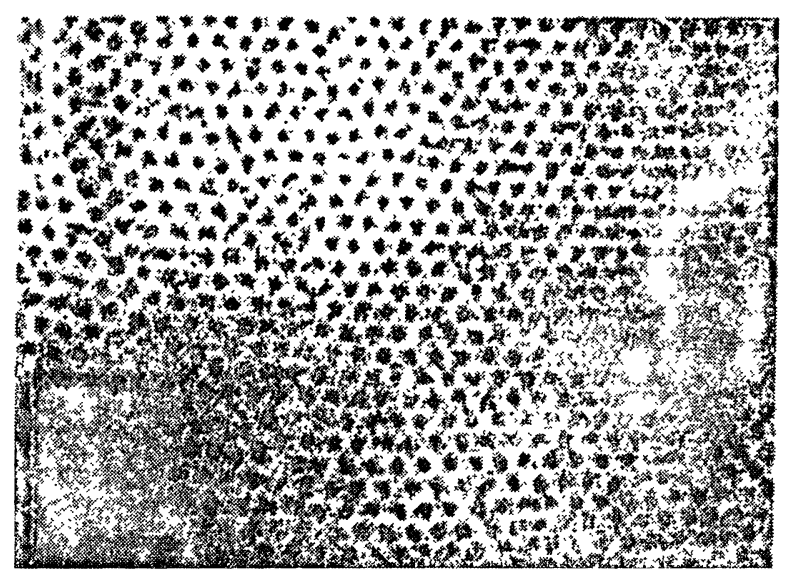

Fig. 2. - Flux line lattice (from Trauble and Essmann).

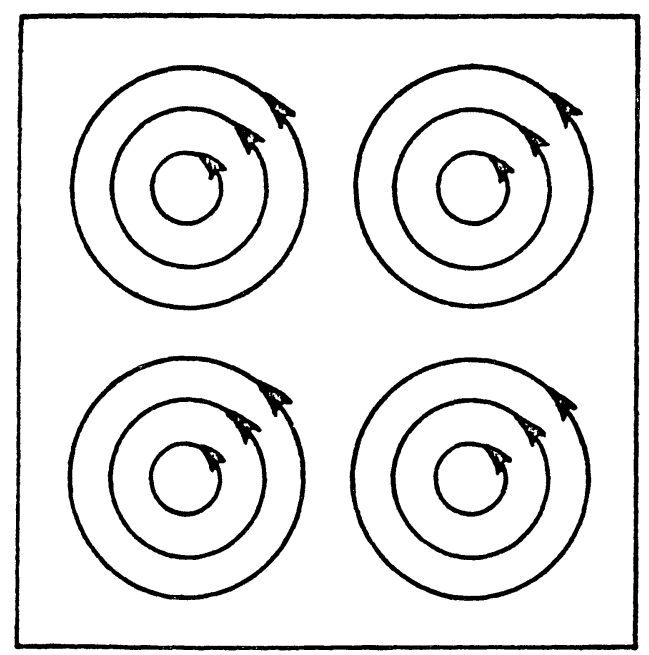

FIG. 3. - Currents around a line of trapped flux.

The trapped flux lines cannot easily be moved in the superconductor because of their tendency to become pinned at grain boundaries, dislocations, strains, etc. It is the existence of these pinning sites which prevents a perfect Meissner effect. The pinning sites can be decreased in number by using very pure material for the shield and by careful annealing of the shield after fabrication. Experiments show however that even though these efforts are made the Meissner effect is still far from complete. Means must be devised to decrease the amount of flux which the Meissner effect must remove from the material. The ambient field must be reduced as low as possible before the shield is made superconducting. After the shield is completely superconducting the ambient field can be raised to any value which does not exceed the critical field at the surface of the shield. The infinite conductivity of the superconductor will keep the interior of the shield field free.

One of the first attempts to get regions of very low magnetic field was an experiment of Mercereau and Vant-Hull in which a superconducting cylinder was spun rapidly while it was cooled slowly through the superconducting transition. Any ambient magnetic fields which do not have axial symmetry with respect to the cylinder will, when seen from the frame of the rotating cylinder, appear to be a time varying field of frequency $\omega$, if $\omega$ is the cylinder rotation frequency. Thus eddy currents will be set up in the rotating cylinder which will attempt to counteract these time varying fields.

If the cylinder is started rotating while warmed above its superconducting transition temperature these eddy currents will tend to exclude or screen the resultant time varying field but because of the finite conductivity will not screen it completely and will decay if the rotation is stopped. Measurements of the electrical resistivity of lead show that the resistivity of the metal drops abruptly above the transition temperature, becoming very small before the metal becomes superconducting. Thus if the cylinder is slowly cooled while rotating the eddy currents induced by the rotation will approach those which would be induced if the cylinder had zero resistance, i.e. they will approach the superconducting screening currents.

As the cylinder makes the transition from the normal to the superconducting state, the superconducting electrons will attempt to exclude any magnetic flux which penetrates the material. Since currents are already flowing which exclude the field which does not have axial symmetry the Meissner currents can be much smaller than they otherwise would have been. Since there is less flux penetrating the cylinder there is a correspondingly lower number of pinned flux lines and thus a much smaller transverse field than there otherwise would have been.

It should be noted that this technique results in a field inside the cylinder which has small transverse components. It will not shield against a field which has axial symmetry with respect to the axis of rotation. Thus it results in a very uniform field along the axis of the cylinder. If the field is also desired to be small, it is necessary to reduce the axial field component by using supplementary coils or high permeability magnetic shielding. This must be done as the shield is cooled because once it is superconducting the flux is trapped in the cylinder and cannot be removed without driving the cylinder into the normal state.

The results of Vant-Hull and Mercereau are shown in figure 4. Qualitatively they agree with the physical explanation given above. The transverse flux excluded from the cylinder is proportional to the rate at which the cylinder is spun. Their results demonstrate that the eddy current shielding is similar enough to 


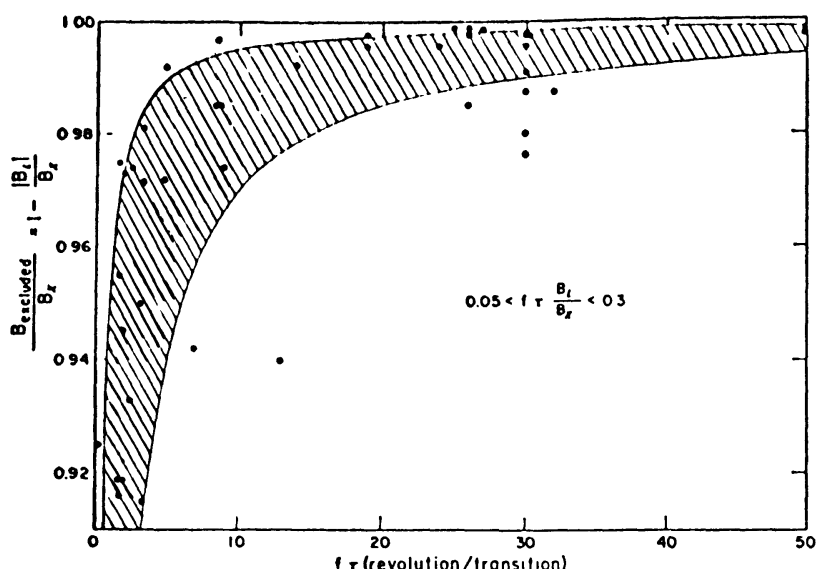

FIG. 4. - Excluded magnetic field vs. number of revolutions made during the transitions from the normal to the superconducting state (from Vant-Hull and Mercereau).

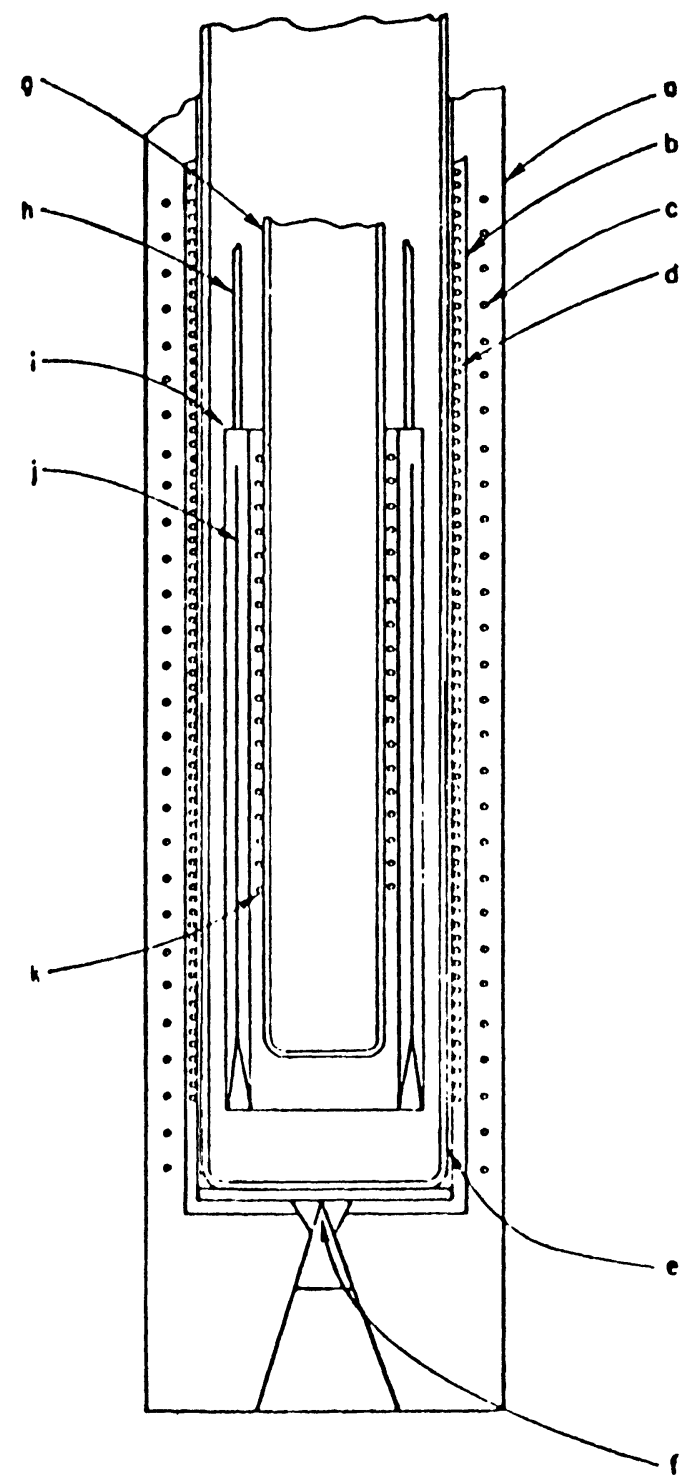

FIG. 5. -- Apparatus for spinning a superconducting shield. The shield is shown by $\mathrm{j}$, its vacuum container is $i$, the rotating bearing is $f$ (from Deaver and Goree).

the Meissner currents which are induced when the cylinder becomes superconducting that these eddy currents decrease the number of trapped flux lines in the shield after the superconducting state is attained. Vant-Hull used tin as the superconductor and controlled the temperature by pumping on the surrounding helium bath. The field was measured with a Hall probe. The difficulty of predicting the exact field configuration is demonstrated by looking at the scatter in the experimental points. The shaded area represents the region in which $60 \%$ of the points were located. Vant-Hull and Mercereau attribute the scatter to uneven cooling and to inhomogeneities in the $1 \mathrm{~mm}$ thick tin cylinder.

Deaver and Goree have used the same techniques as Vant-Hull and Mercereau. Their apparatus is shown in figure 5. They have also measured the attenuation of an external axial field as one moves down in the cylinder. Their results are shown in figure 6 . They find that the axial magnetic field decreases as :

$$
H=H_{0} \mathrm{e}^{-3 \cdot 4 z / r}
$$

which is in fair agreement with the value one obtains theoretically by treating the superconducting shield as a substance which is completely impermeable to the applied magnetic field.

Measurements on the shielding by a diamagnetic superconducting cylinder on a transverse magnetic field were also performed by Deaver and Goree but

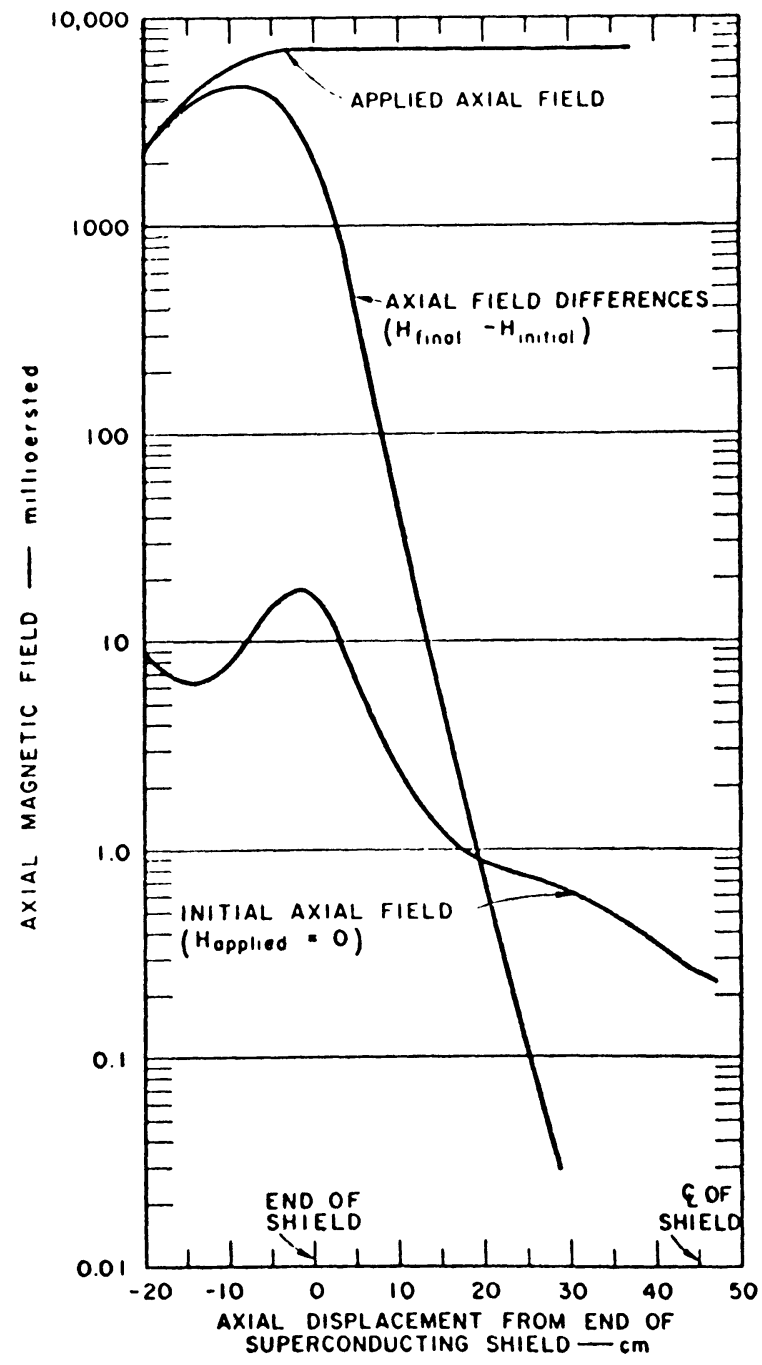

Fig. 6. - Shielding by a cylinder from a small externally applied axial field (from Deaver and Goree) 
no quantitative measurements were taken. They found that the transverse field fell off much less rapidly however as they went down into the cylinder. The theoretical attenuation should go as :

$$
H=H_{0} \mathrm{e}^{-\frac{1.8 z}{R}}
$$

a factor of 6 per radius. We have found approximate agreement with this law in measurements performed at Stanford but because our shields are not perfect cylinders no quantitative data has been taken.

The effect of high transverse magnetic fields has been demonstrated by de Haas and many others. The data in figure 7 are taken from a paper by Gittle-

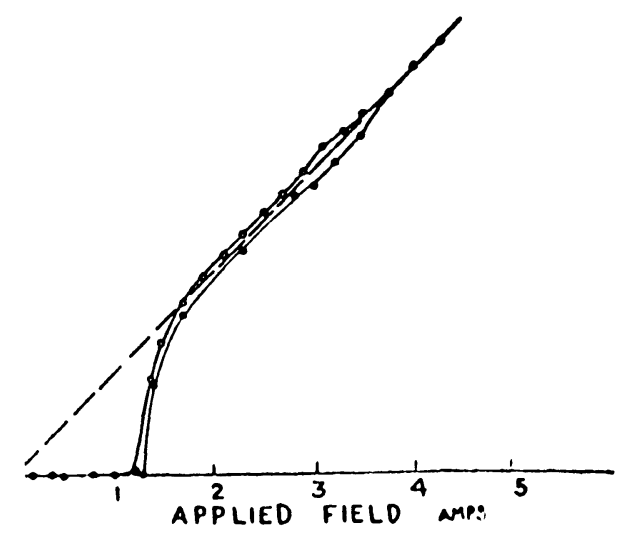

FIG. 7. - Field inside a superconducting cylinder vs. externally applied field (from Gittleman).

man and are typical. He measures the transverse field at the center of a tin cylinder and increases the external field. The cylinder is not rotated. There is perfect shielding within the detection capabilities of his magnetometer until a threshold is reached. At that point the field begins to penetrate the cylinder. The percentage of field penetration increases until the transverse field inside the cylinder equals the applied field outside and a large distance away. Gittleman's model of how the flux penetrates the cylinder is shown in figure 8 . It is in good agreement with the modern picture described earlier. If the external field is decreased after the magnetic flux has begun to penetrate, the magnetic behavior of the cylinder is non reversible and the flux remains trapped. Since the field must reach the critical field somewhere on the sample in order for the flux to begin to penetrate, the importance of keeping the field incident on the super-

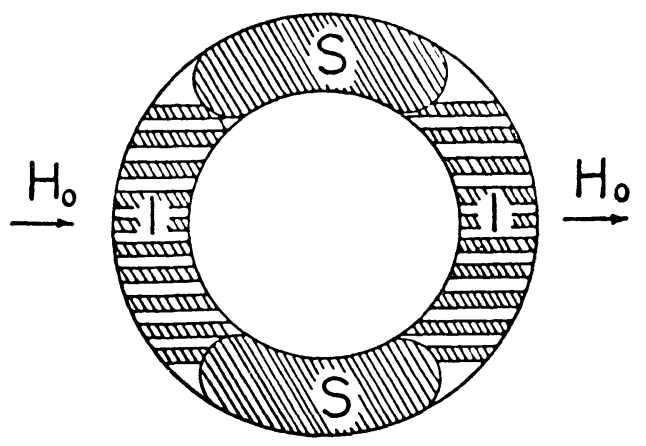

FIG. 8. - Intermediate state of a superconducting cylinder after flux has penetrated (from Gittleman). conducting shield as low as possible cannot be over emphasized.

It has been pointed out before that the Meissner effect has never been seen to be perfect. Bol discovered that the amount of flux excluded from metal cylinders which were being spun depended on such things as the amount of strain in the metal. He found that this strain appeared even at low temperatures because of the centrifugal forces arising from spinning. Thus, by itself, it is probably not sufficient to depend on the Meissner effect to obtain an extremely low field. However there are other schemes which utilize the tendency of a superconductor to exclude flux and the phenomenon of flux quantization which in principle are capable of lowering the field even to zero.

One such method is illustrated in figure 9 . If a

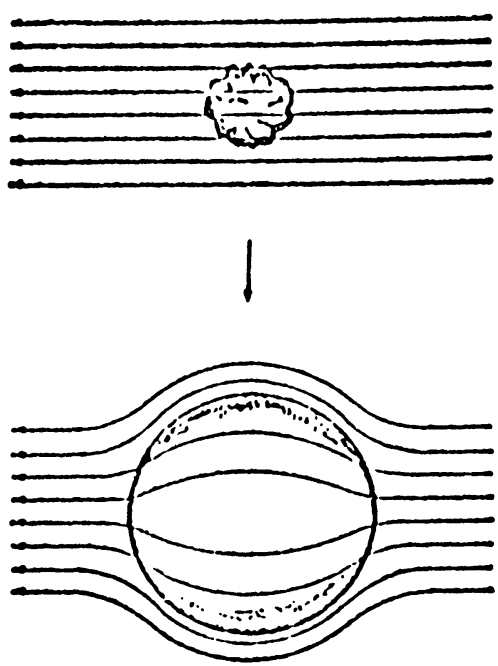

FIG. 9. - Field reduction

by a superconducting bladder.

flexible superconducting bladder is cooled in a given magnetic field, it will exclude some of the flux incident on the bladder and trap the remainder. If the bladder is then unfolded, the density of flux lines inside the bladder can be much lower, hence the field is lower. Note that from inside the bladder a trapped flux line looks approximately like a small current loop. If another bladder is then inserted inside the first and cooled slowly enough it should trap less flux than the first because it has cooled in a lower magnetic field. The second bladder can then be expanded, thus decreasing the flux still further and a third bladder inserted inside the second. Thus in principle, if the experimenter is careful enough, it should be possible to decrease the field indefinitely.

There will come the point where the field on an unopened, non superconducting bladder will be low enough that the flux linked by the bladder is less than one half a flux unit. At this point, when the bladder becomes superconducting, it is energetically favorable for it to exclude all of the flux. Thus when that bladder is expanded there should be zero magnetic field inside. The use of the quantum condition has allowed us to suddenly switch from talking about decreasing the field to talking of making it absolutely zero.

Brown has published his efforts to obtain field reductions by superconducting bladder expansions. $\mathrm{He}$ 
used commercially available lead foil $.07 \mathrm{~mm}$ thick. The lead was melted to make the bladder seam using a gas flame and no flux. No solder material other than the lead itself was used. Brown's bladders were long cylinders of about $11 \mathrm{~cm}$ diameter and $35 \mathrm{~cm}$ length. These bladders were folded by pleating them into pleats of about $1 \mathrm{~cm}$ width. No folding was done to decrease the length, thus they should exclude and reduce the field along the axis of the cylinder. The decrease in the component of the field perpendicular to the axis is geometrical in nature and depends on the particular pattern of folding.

Brown measured his magnetic field with the flux gate magnetometer discussed in this conference by Edwards. He measured a field reduction by a factor of 500 in each balloon expansion but did not check the field below $10^{-6}$ gauss because of the instrument used. Brown also checked the attenuation of external d.c. fields inside his bladders and found the decrease to be exponential. His results are shown in figures 10 and 11 .

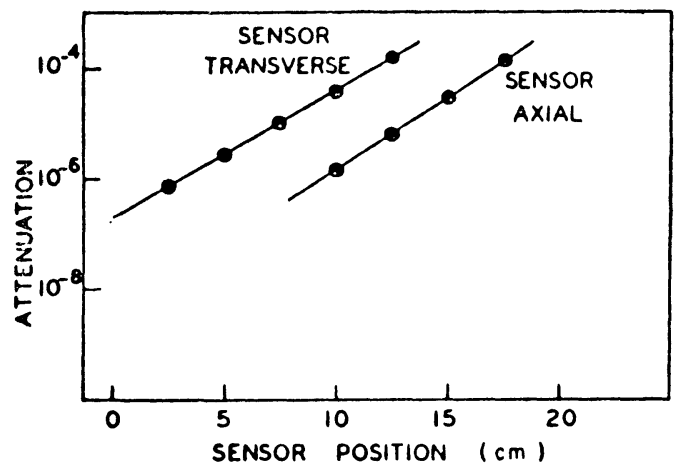

FIG. 10. - Attenuation of a small external transverse field (from Brown).

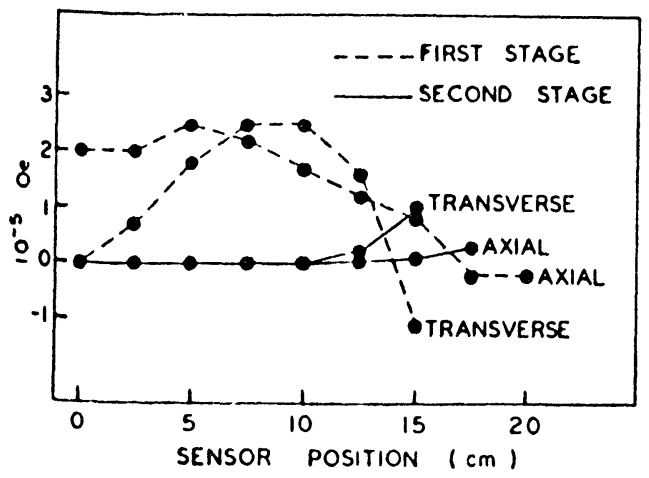

FIG. 11. - Field profile

in a superconducting bladder (from Brown).

Probably the most complete study of the properties of superconducting bladders has been conducted at Stanford. This work was begun by Varoquaux and Rose and has been continued by Cabrera. Cabrera has examined the attenuation which is obtained due to the Meissner effect only as well as the geometrical attenuation due to the folding configuration of the bladder. He has used the double point contact junction discussed at this conference by de Bruyn-Ouboter. His device had a sensitivity to magnetic fields of at least $10^{-9}$ gauss. He also included a superconducting flip-coil which transformed the field to his point- contact device and so had the capacity to examine the direction and magnitude of the magnetic field at any point accessible to the coil. He could also obtain an estimate of the homogeneity of the field by examining the current in his coil as it was rotated past a certain area of the lead bladder.

Cabrera's measurements indicate that for a rolled lead bladder the Meissner effect will cause a reduction in the flux density by a factor of about 100 . In other words, if a lead bladder is flattened and rolled then cooled the field inside the bladder after unrolling will be less by a factor of about 100 . However, experimentally there seems to be a lower limit of about $10^{-6}$ gauss. It is found that a second bladder rolled and cooled in an identical manner inside the first will not further reduce the field.

Cabrera has also examined the field reduction which can be obtained by folding the balloon in such a manner as to obtain cancellation of the effects of different areas of trapped flux. Brown obtained his field reductions in bladders which were pleated about 12 times, Cabrera tested a simple double pleated arrangement. In the low field region, i.e. in fields less than $10^{-5}$ gauss, Cabrera finds very little reduction due to the folding and he also finds a very inhomogeneous field. We will discuss the reasons for the poor field reduction later.

The inhomogeneity of the field is demonstrated in figure 13. The flip coil of Cabrera's magnetometer is rotated through $180^{\circ}$ and the magnetometer pickup is plotted. The double point contact magnetometer is set up to read fields modulo one flux unit and the large variations indicate the change in the critical current of the device as the flux increases through several flux units. This type of behavior is expected whenever the field is larger than that corresponding to one flux unit through the coil. The inhomogeneity is best illustrated by the small field reversals which occur between the points marked $90^{\circ}$ and $180^{\circ}$. It is evident that as the edge of the flip coil traverses a certain region the field is subject to rapid sign reversals : on the order of $10^{-7}$ gauss reversals in several millimeters travel. This inhomogeneity is probably due to the coil's nearing a region containing a large amount of trapped flux.

We must now ask for the reasons why the field does not decrease below the $10^{-6}$ gauss region in the second and further expansions. This question is not completely answered and further experiments are in progress to determine it. At this time the most reasonable explanation seems to be that magnetic flux is trapped by the thermal currents which are induced as the lead is cooled through its transition temperature. This phenomenon is probably also responsible for the large scatter seen in the data of Mercereau and Vant-Hull. The mechanism is that as the lead is cooled, temperature gradients will be induced in the material and as the temperature is changed Thomson currents will flow. These currents can be quite large if the resistance of the metal is small. Since the resistance of most materials is observed to drop abruptly above the superconducting transition temperature it is not unlikely that large currents will flow if the material is not cooled slowly enough. These currents will then be trapped if the material goes into the superconducting state before they decay due to ohmic losses. Preliminary experiments in our laboratory and experiments discussed by Hildebrand at this conference indicate that 


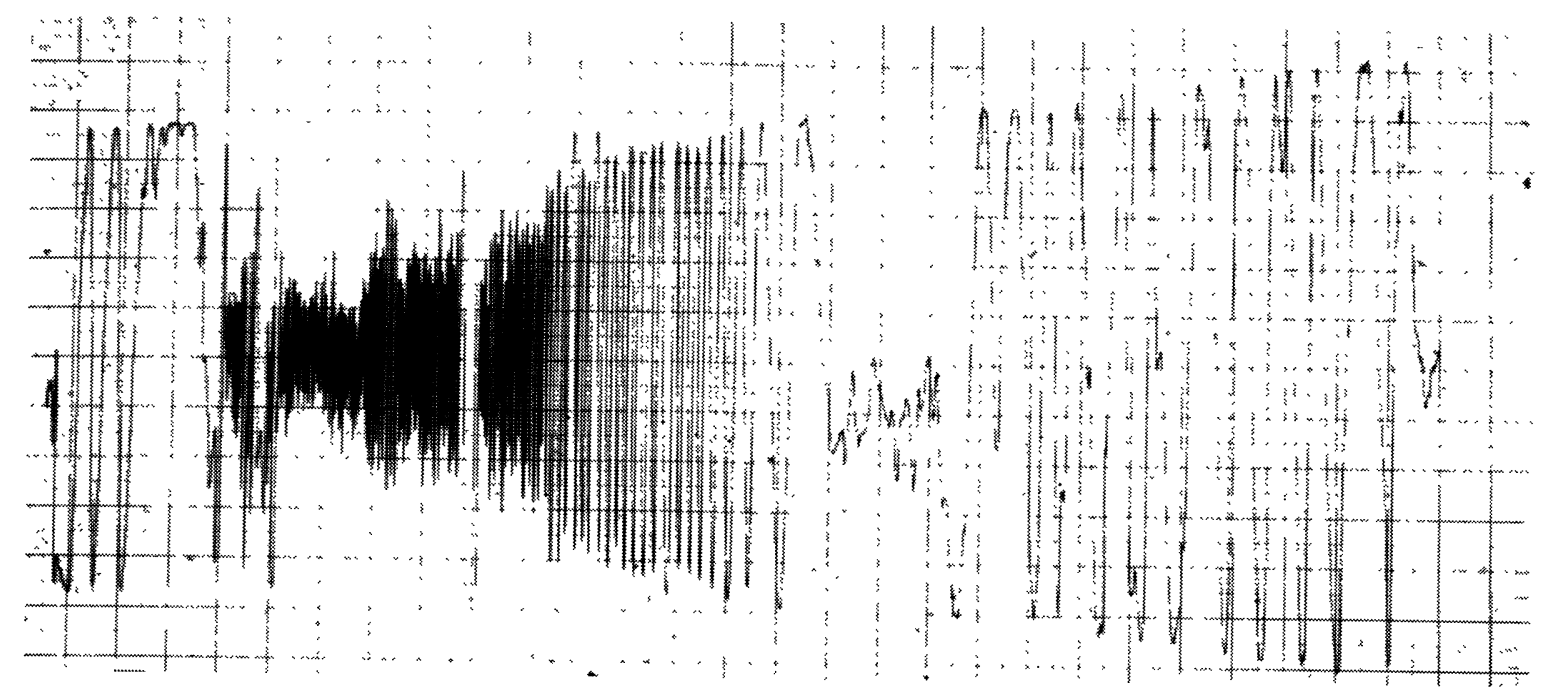

FIG. 12.

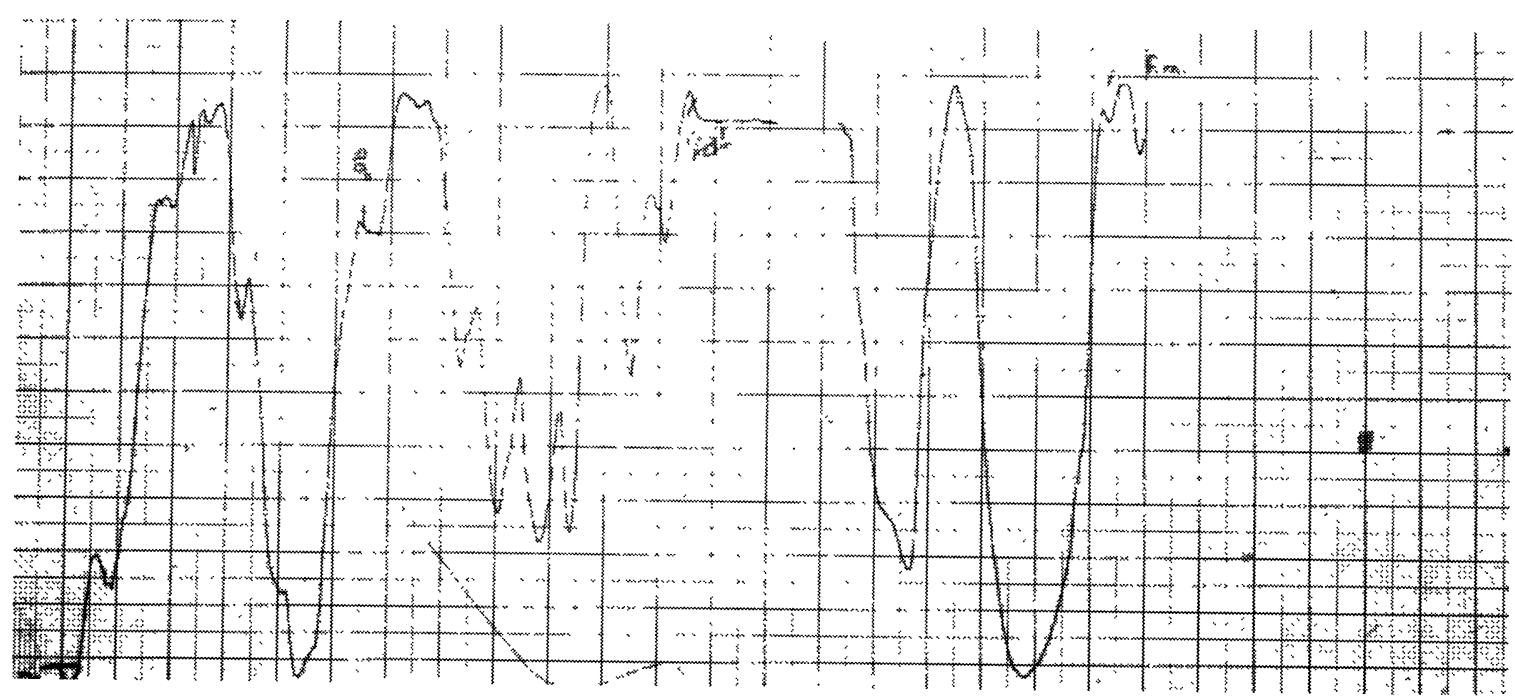

FIG. 13.

the effects of thermal currents can be eliminated by slow cooling.

Professor Fairbank has suggested that slow cooling coupled with a sharp temperature gradient may be the best method to eliminate trappedflux, both external and thermally generated. He calls his technique "heat flushing". The idea is that if one can keep the area of the region which is near the transition temperature small enough then, because of flux quantization, it will be impossible for the material to trap any flux. The technique is then to keep a sharp temperature gradient on the material right above the liquid helium and to let the helium rise over the material slowly so as to keep the currents small. Then if the temperature gradient is sharp enough so that the area which is near the transition temperature is small enough and regular then it will be energetically unfavorable for the material to trap any magnetic flux. Inside the shield then there should be a region with no trapped flux and if the shield is closed there will be no magnetic field.

The effects of heat flushing are dramatically shown in figure 12, recorded by Cabrera. Here the helium level was allowed to rise slowly over a tightly rolled bladder. It was intended that the bladder should cool from the top down, thus heat flushing the magnetic flux toward the bottom. Near the top of the bladder after flushing and expansion the field was $2 \times 10^{-7}$ gauss, a reduction by a factor of 10 . The magnetometer was then lowered from this point down into the bladder and the response recorded. We see that flux was flushed downward somewhat but that the flux was apparently trapped by the superconductivity, probably because the bottom end of the bladder also became cold and flushed flux upward. The trapped flux is concentrated about 14 inches from the bottom of the bladder and at that point the field was about $10^{-5}$ gauss, a factor of 10 higher than the original field before the bladder was cooled. This figure illustrates that the concept does work and also points out where care must be taken in using it.

V. Applications. - It is clear that many new fundamental experiments can make use of the environment which one can obtain with superconducting shielding. One such experiment is that designed to 
search for a permanent electric dipole moment in the ${ }^{3} \mathrm{He}$ nucleus. This experiment is now underway at Stanford University.

A sample of liquid ${ }^{3} \mathrm{He}$ is aligned by optical pumping and placed at the center of a superconducting sphere from which the last quantum of trapped flux has been excluded. Since it is in a region of zero magnetic field the spin will remain pointing in a fixed direction, there being no torques applied to the nuclear spin. If now a strong electric field is applied to the sample by capacitor plates inside the shield a torque will be put on the nucleus if it has a permanent electric dipole moment. This torque will cause the nuclear spin to precess about the electric field. This precession will be detected by the superconducting magnetometer discussed by Fairbank and by Opfer in this conference.

This experiment requires that the electric and magnetic environment of the sample remain constant for many days. Such stability is guaranteed by the use of superconducting shielding. It requires an absolutely zero magnetic field which is attainable only by a superconductor. Thus the possibility of using this shielding allows an experiment to be considered which otherwise would have been considered to be impossible.

\section{REFERENCES}

BoL (M.), Ph. D. Thesis, Stanford University, 1965. Brown (R. E.), Rev. Sci. Instr., 1968, 39, 547.

Deaver (B. S.) and FaIrbank (W. M.), Phys. Rev. Lett., 1961, 7, 43.

DEAVER (B. S.) and GoreE (W. S.), Rev. Sci. Instr., 1967, 38, 311

Doli (R.) and Naubauer (M.), Phys. Rev. Letters, 1961, 7, 51 .

Giturleman (J.), Phys. Rev., 1953, 92, 561.
LoNDON (F.), Superfluids, I (Dover Publications, New York, 1961).

MEISSNER (W.) and OCHSENFELD (R.), Naturwissenshaften, 1933, 21, 787.

Trauble (H.) and Essmann (V.), J. Appl. Phys., 1968, 39, 4052.

Vant-Huli (L. L.) and Mercereau (J. E.), Rev Sci. Instr., 1963, 34, 1238. 